\section{All-birefringent-fiber frequency-modulated continuous-wave Sagnac gyroscope}

\author{
Jesse Zheng \\ PhotonTech \\ 1980 E51 Avenue \\ Vancouver, British Columbia, V5P 1 V9 \\ Canada \\ E-mail: gang.zheng@aicompro.com
}

\begin{abstract}
An all-birefringent-fiber frequency-modulated continuous-wave (FMCW) Sagnac gyroscope is described. The rotation velocity of the gyroscope is determined by measuring the phase shift of the beat signal produced by the two counter-propagating laser beams in the birefringent fiber coil. A resolution of $0.02 \mathrm{deg} / \mathrm{sec}$ with a $100-\mathrm{m}$ fiber coil has been observed. () 2005 Society of Photo-Optical Instrumentation Engineers.
\end{abstract}

[DOI: $10.1117 / 1.2000987]$

Subject terms: fiber optic sensors; gyroscopes; diode lasers.

Paper 050207 LR received Mar. 17, 2005; revised manuscript received Jun. 20, 2005; accepted for publication Jun. 20, 2005; published online Aug. 10, 2005.

Recently, the interest in optical frequency-modulated continuous-wave (FMCW) fiber-optic gyroscopes restarted. ${ }^{1-3}$ Optical FMCW interference, a new technology derived from radar, has a number of advantages, including easy calibration of the fractional phase, easy determination of the phase-shift direction, easy count of the full periods, and absolute measurement. ${ }^{4-6}$ The application of optical FMCW interference to rotation sensing not only can solve the problems in the conventional fiber-optic gyroscopes, such as zero-sensitivity point, inaccurate phase calibration, ambiguous shift direction determination, and $\pm \pi / 2$ phase shift restriction, but also can reduce the size and weight because the fiber-optic FMCW gyroscope does not use bulk phase modulators or frequency shifters.

Fiber-optic FMCW Sagnac gyroscopes have to be unbalanced so that the beat signal with a proper frequency can be obtained. This requirement normally makes fiber-optic FMCW gyroscopes complicated. However, if a fiber-optic FMCW gyroscope uses a birefringent fiber coil, its structure will be very simple because a single length of birefringent fiber can be used as a double-beam unbalanced interferometer if the light is coupled into its two orthogonal polarization modes.

Figure 1 shows schematically an all-birefringent-fiber FMCW gyroscope, which consists of a single-mode laser diode with a fiber pigtail, a fiber-optic polarizer (FP), a polarization-maintaining fiber coupler (PMFC), a birefringent fiber coil, and a photodetector. The output fibers of the PMFC are connected with the birefringent fiber coil in the same polarization directions, but the coordinates of the principal axes on the two ends of the birefringent fiber coil have a 90 -deg rotation.
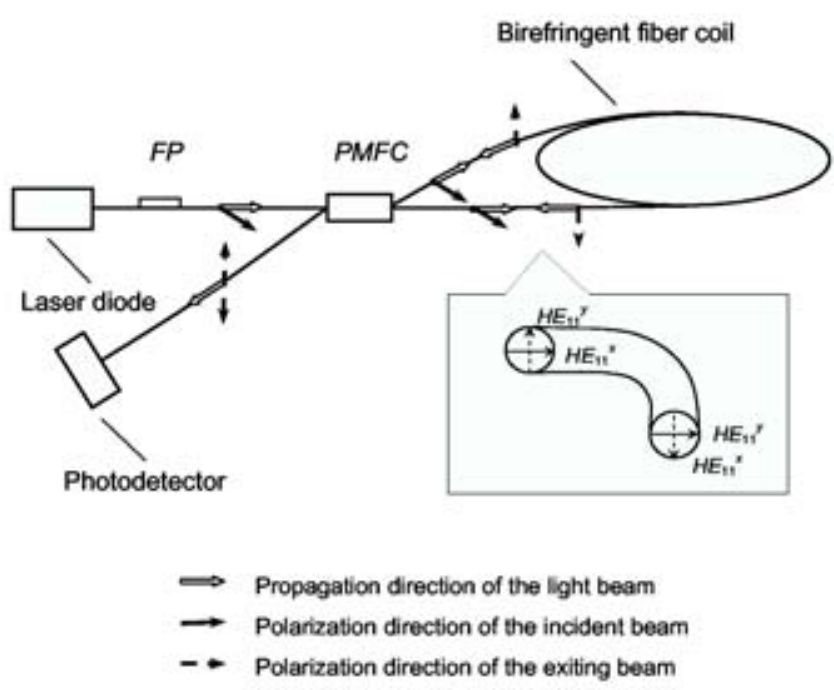

Fig. 1 All-birefringent-fiber FMCW Sagnac gyroscope.

A linearly polarized FMCW laser beam is first coupled into one input fiber of the PMFC in one polarization mode (say the $\mathrm{HE}_{11}^{x}$ mode) and divided into two beams. These two beams are then coupled into the birefringent fiber coil in different polarization modes from the different ends. For instance, the clockwise propagating beam is coupled into the $\mathrm{HE}_{11}^{x}$ mode, while the anti-clockwise propagating beam is coupled into the $\mathrm{HE}_{11}^{y}$ mode. After traversing the fiber coil, these two beams are recombined by the PMFC, and the beat signal is detected by the photodetector.

Because the polarization direction of each beam rotates 90 deg after emerging from the birefringent fiber, the polarization directions of the two exiting beams are still parallel to each other, but they are perpendicular to the polarization direction of the incident beams. This property is very useful, because it ensures that the best signal contrast is always obtained.

The initial optical path difference $O P D$ between the two counter propagating beams in the birefringent fiber coil can be written as

$O P D=\left(n_{e x}-n_{e y}\right) L$,

where $n_{e x}$ is the effective refractive index of the $\mathrm{HE}_{11}^{x}$ mode, $n_{e y}$ is the effective refractive index of the $\mathrm{HE}_{11}^{y}$ mode, and $L$ is the total length of the birefringent fiber coil.

The signal of optical FMCW interference has been systematically analyzed by the author elsewhere. ${ }^{6}$ If the laser frequency is modulated with a sawtooth waveform, the intensity of the beat signal in a modulation period $I(t)$ can be written as

$$
\begin{aligned}
I(t) & =I_{0}\left[1+V \cos \left(\frac{2 \pi \Delta v v_{m} O P D}{c} t+\frac{2 \pi}{\lambda_{0}} O P D\right)\right] \\
& =I_{0}\left[1+V \cos \left(2 \pi v_{b} t+\phi_{b 0}\right)\right],
\end{aligned}
$$

where $I_{0}$ is the average intensity of the beat signal, $V$ is the contrast of the beat signal, $\Delta \nu$ is the optical frequency modulation excursion, $\nu_{m}$ is the modulation frequency, $c$ is the light speed in free space, $\lambda_{0}$ is the central optical wavelength in free space, and $\nu_{b}$ and $\phi_{b 0}$ are the frequency and

0091-3286/2005/\$22.00 @ 2005 SPIE 
initial phase of the beat signal, respectively $\left(\nu_{b}\right.$ $\left.=\Delta \nu \nu_{m} O P D / c, \phi_{b 0}=2 \pi O P D / \lambda_{0}\right)$.

When the birefringent fiber coil rotates around its vertical axis, the Sagnac phase shift $\delta \phi_{b 0}$ in the beat signal can be written as

$\delta \phi_{b 0}=\frac{4 \pi R L \Omega}{C \lambda_{0}}$,

where $R$ is the radius of the birefringent fiber coil and $\Omega$ is the rotation angular velocity. Therefore, the rotation angular velocity $\Omega$ is equal to

$\Omega=\frac{c \lambda_{0}}{4 \pi R L} \delta \phi_{b 0}=k \delta \phi_{b 0}$

where $k$ is the scale factor of the birefringent fiber FMCW gyroscope in $\mathrm{sec}^{-1}$, given by

$k=\frac{c \lambda_{0}}{4 \pi R L}$.

The experimental arrangement consists of a single longitudinal mode laser diode lasing at $660 \mathrm{~nm}$, a silicon p-i-n photodiode with 1-ns rise time, and an elliptical-core birefringent fiber coil of $100 \mathrm{~m}$ in length and $0.1 \mathrm{~m}$ in radius. The optical frequency of the laser diode is modulated with a sawtooth waveform at a frequency of $10 \mathrm{KHz}$, by modulating its driving current. The polarization axes of the fiber coil and the leading fibers of the PMFC are carefully determined and marked on the fiber jacket, and then the fiber coil and PMFC are connected according to the requirement shown in Fig. 1. The signal detected is first sent to an auto-gain amplifier to minimize the affection of light power, and then sent to a bandpass filter to choose the most intensive harmonic component of the beat signal to compare phase with a standard sinusoidal reference signal of the same frequency. The reference signal is obtained by first transforming the waveform of the laser driving current from a sawtooth wave to a sinusoidal wave, and then multiplying the frequency of this sinusoidal wave. Figure 2 indicates the relationship between the rotation angular velocity of the fiber coil and the phase shift of the beat signal. The experimental results show that a resolution of $0.02 \mathrm{deg} / \mathrm{sec}$ can be obtained.

The accuracy of the gyroscope is mainly limited by the nonreciprocal phase drift of the birefringent fiber coil and the frequency drift of the laser diode. The former is due to the thermal expansion of the birefringent fiber coil, the lat-

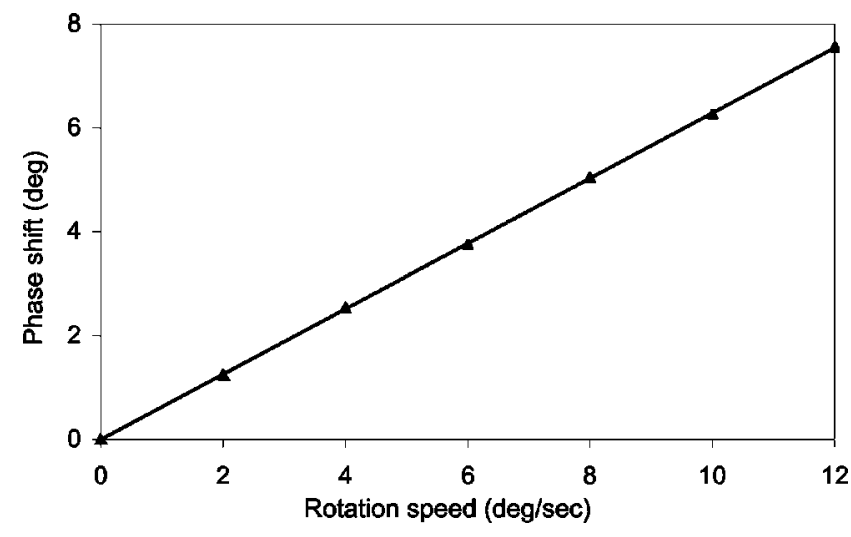

Fig. 2 Relationship between the rotation angular velocity of the birefringent fiber coil and the phase shift of the beat signal.

ter is due to the effect of the temperature on the laser diode. The expansion of the birefringent fiber coil increases the optical path lengths of the two counter-propagating beams differently, because the effects of temperature on the two polarization modes are not the same. Therefore, in order to improve the accuracy and long-term stability, a proper temperature control system for both the laser diode and the fiber coil should be employed.

In summary, the first all-birefringent-fiber FMCW gyroscope has been described. The advantages of this fiber-optic gyroscope include high accuracy, long dynamic range, small size, no polarization noise, and full passiveness. The limitation is that the birefringent fiber coil may introduce an unexpected nonreciprocal phase drift if the environmental parameters (such as temperature) change, and therefore, a proper temperature control system should be used in the practical situation.

\section{References}

1. B. Culshaw and I. P. Giles, "Frequency modulated heterodyne optical Sagnac interferometer," IEEE J. Quantum Electron. QE-18, 690-693 (1982).

2. J. Zheng, "Singlemode fibre frequency-modulated continuous-wave Sagnac gyroscope," Electron. Lett. 40, 1255-1257 (2004).

3. J. Zheng, "All-fiber single-mode fiber frequency-modulated continuous-wave Sagnac gyroscope," Opt. Lett. 30, 17-19 (2005).

4. D. A. Jackson, A. D. Kersey, M. Corke, and J. D. C. Jones, "Pseudoheterodyne detection scheme for optical interferometers," Electron. Lett. 18, 1081-1083 (1982).

5. D. Uttam and B. Culshaw, "Precision time domain reflectometry in optical fiber systems using a frequency modulate continuous wave ranging technique," J. Lightwave Technol. LT-3, 971-976 (1985).

6. J. Zheng, "Analysis of optical frequency-modulated continuous-wave interference," Appl. Opt. 43, 4189-4198 (2004). 